\title{
ASPECTOS ECOLÓGICOS DE DIAPHORINA CITRI KUWAYAMA, 1908 EN EL CULTIVO DE CÍTRICOS EN VILLA ALTAGRACIA Y EL SEIBO, REPÚBLICA DOMINICANA
}

\author{
Domingo Josué Henríquez Liria ${ }^{1}$, Santo Navarro Morales ${ }^{2}$, Ruth H. Bastardo ${ }^{2}$ \\ y Modesto Reyes Valentín ${ }^{3}$
}
${ }^{1}$ Escuela de Biología, Facultad de Ciencias, Universidad Autónoma de Santo Domingo, Santo Domingo, República Dominicana. dracomyrmex@yahoo.com.
${ }^{2}$ Instituto de Investigaciones Botánicas y Zoológicas Prof. Rafael M. Moscoso, Facultad de Ciencias, Universidad Autónoma de Santo Domingo, Santo Domingo, República Dominicana. botanicayzoo@uasd.edu.do.
${ }^{3}$ Facultad de Ciencias Agronómicas y Veterinarias, Universidad Autónoma de Santo Domingo, Santo Domingo Oeste, RepúblicaDominicana.fcav@uasd.edu.do.

\section{RESUMEN}

Se determinó la distribución espacial y dinámica de la plaga Diaphorina citri Kuwayama, 1908 (Hemiptera: Psyllidae) en plantaciones de cítricos en los municipios de Villa Altagracia, provincia San Cristóbal y en La Candelaria, paraje Los Corazones, provincia de El Seibo. En Villa Altagracia, D. citri resultó con patrón agregado para los inmaduros y patrón uniforme en los adultos; los picos poblacionales más altos de $D$. citri ocurrieron en febrero, marzo, mayo y agosto. En El Seibo, los inmaduros de D. citri registraron patrón uniforme y los adultos agregación; en abril y junio se presentó mayor actividad de las poblaciones. En Villa Altagracia se registró una mayor densidad poblacional que en El Seibo. En cada localidad, las fases fenológicas con mayor densidad poblacional fueron la fructificación y la brotación vegetativa. La disposición espacial, las fluctuaciones y densidades poblacionales, siempre estuvieron relacionadas con la densidad y disponibilidad de brotes tiernos en cada cultivo. En las zonas de estudio, se observó baja correlación entre la temperatura y las lluvias registradas con la plaga, no obstante, se observó gran actividad poblacional de $D$. citri en períodos con temperatura y lluvias favorables para la misma.

Palabras clave: ecología, psílido asiático, disposición espacial, cítricos, fenología.

Title: Ecological aspects of Diaphorina citri Kuwayama, 1908, in citrus groves in Villa Altagracia and El Seibo, Dominican Republic.

\section{ABSTRACT}

In this study the spatial distribution and dynamics of the pest Diaphorina citri Kuwayama, 1908 (Hemiptera: Psyllidae) in citrus groves in Villa Altagracia and La Candelaria, Los Corazones, El Seibo was determinated. According to our results, the immature stages of $D$. citri were found to be aggregated in a spatial pattern while the adults were found in a uniform pattern. In El Seibo, the distribution of eggs and nymphs of $D$. citri showed uniformity and in adults the distribution was aggregated. In Villa Altagracia, the highest population peaks occurred in February, March, May and August, while in El Seibo, in April and June. The highest population density was always found in Villa Altagracia during the time of the experiments more than it was in El Seibo. Phenological phases such as fruiting and budding were the most densely populated. In both places, the spatial fluctuations and population densities were always related to the density and availability of young shoots in each culture. Temperature and rains registered in the study areas had a low correlation with the plague, however, population peaks were observed in periods of favorable weather conditions for this insect.

Keywords: ecology, Asian psyllid, spatial arrangement, citrus, phenology. 


\section{INTRODUCCIÓN}

La especie Diaphorina citri Kuwayama, 1908 (Hemiptera: Psyllidae) es el vector más importante de la bacteria Candidatus Liberibacter asiaticus, que produce la enfermedad conocida como el Huanglongbing (HLB) o enverdecimiento de los cítricos (Capoor et al., 1967; French et al., 2001; Michaud, 2002; Halbert y Manjunath, 2004; Qureshi y Stansly, 2007; Ramos Méndez, 2008; Hall et al., 2008; Moreno Pérez et al., 2008; Cabrera Mireles y Murillo Cuevas, 2010; Barrera et al., 2011). Este es el complejo vector-patógeno que más afecta los cultivos de cítricos en todo el mundo (Villalobos et al., 2005; Polek et al., 2007; Morales et al., 2010).

En la República Dominicana, las pérdidas anuales por los costos en el manejo del complejo D. citri-HLB son de RD $\$ 295$ millones. Debido al impacto negativo ocasionado por el complejo D. citri - HLB, muchos productores, principalmente pequeños, tuvieron que eliminar muchas plantas al detectarse este complejo en sus campos. La incidencia de D. citri y el HLB en los cultivos de cítricos, provocó grandes pérdidas o la quiebra para muchos productores.

\section{OBJETIVO}

- Contribuir al conocimiento de la ecología y dinámica de $D$. citri en los agroecosistemas citrícolas dominicanos para favorecer el desarrollo e implementación de prácticas compatibles con el manejo integrado de esta plaga.

\section{MATERIALES Y MÉTODOS}

Esta investigación se realizó en el municipio de Villa Altagracia, provincia San Cristóbal y en La Candelaria, paraje Los Corazones, provincia de El Seibo, en cultivos de Citrus sinensis (L.) Osbeck, variedad naranja "Valencia", desde enero hasta agosto del 2013. Se seleccionaron 50 árboles al azar, 25 plantas fijas en cada localidad. Se distribuyeron los árboles en grupos de 5, con 5 plantas cada uno; se evaluó la planta central y los 4 árboles alrededor de esta en la dirección de los cuadrantes cardinales, norte, sur, este y oeste. De cada árbol se tomaron 4 brotes tiernos, para un total de 100 brotes nuevos en cada observación. En cada localidad las observaciones fueron efectuadas cada 15 días. Se contabilizó el número total de individuos (huevos, ninfas y adultos) presentes en cada brote, el tamaño de los brotes colectados no excedió los $6 \mathrm{~cm}$ de longitud, siguiendo lo propuesto por diferentes autores (Dharajothi et al., 1989; Sétamou et al., 2008; García Darderes, 2009a; Robles-Gonzales et al., 2010; Sandoval-Rincón et al., 2010). Además, se utilizaron trampas amarillas pegantes para monitorear adultos (Hall, 2009; Stansly et al., 2010; Barrera et al., 2011) que no pudieron ser atrapados durante las evaluaciones.

Con los resultados de los conteos de las poblaciones de D. citri, se calculó la media $(\overline{\mathrm{X}})$ y la varianza (S2) de cada etapa del psílido en cada observación; con estas medias se obtuvo una media y varianza global de las fases del insecto. La distribución espacial de D. citri quedó determinada siguiendo la relación entre la varianza y la media de la densidad poblacional (Elliott, 1977, citado por Toledo e Infante, 2008), donde si S2 = X , la distribución es al azar, si S2 $<\overline{\mathrm{X}}$, es uniforme y si S2 $>\bar{x}$, la distribución es agregada. Para tener mayor confiabilidad en los patrones de distribución que presentó la plaga en este estudio, se aplicaron los siguientes índices de agregación: la razón varianza/media (S2/x) o Índice de Dispersión (ID), el coeficiente de Green $\left.(\mathrm{Cx})=(\mathrm{S} 2 / \overline{\mathrm{X}})-1 / \sum \mathrm{x}-1\right)\left(\right.$ Green, 1966), la media de agregación de Lloyd $\left(\mathrm{m}^{*}=\overline{\mathrm{x}}+(\mathrm{S} 2 / \overline{\mathrm{x}})-1\right)$ y el índice de Lloyd $(\mathrm{L}=\mathrm{m} * / \overline{\mathrm{X}})$ (Lloyd, 1967). Paso previo al cálculo de la media, la varianza y a la aplicación de estos índices, los datos, se transformaron por medio de $\sqrt{X+0.5}$ para así obtener una mejor estabilización de la varianza de los mismos (Yamamura, 1999). 
Para establecer las densidades poblacionales de $D$. citri y la fenología del cultivo, se colectaron y contabilizaron las diferentes etapas del desarrollo del insecto quincenalmente durante las fases de brotación-vegetativa, brotación-floral y fructificación en ambas localidades. A los datos se les aplicó la prueba de Shapiro-Wilks $(\alpha=0.05)$ para determinar la normalidad de los mismos, y el resultado de esta prueba mostró evidencias de no normalidad en los datos obtenidos, se aplicó el análisis de varianza no paramétrica de Kruskal-Wallis $(\alpha=0.05)$. Se estableció la relación entre las poblaciones $D$. citri con temperatura y lluvia, por medio del coeficiente de correlación de Spearman y un Análisis de Componentes Principales (ACP), con el objetivo de obtener mejor interpretación de los datos.

El conteo y análisis de las muestras colectadas se realizó en el Instituto de Investigaciones Botánicas y Zoológicas Prof. Rafael M. Moscoso (IIBZ) de la Universidad Autónoma de Santo Domingo. Todas las pruebas estadísticas de esta investigación, fueron realizadas usando el paquete estadístico INFOSTAT versión 2008 (Universidad Nacional de Córdoba, Argentina) y el paquete Microsoft Excel, 2007. Los datos climatológicos fueron facilitados por la Oficina Nacional de Meteorología (ONAMET) de la República Dominicana.

\section{RESULTADOS Y DISCUSIÓN}

Distribución espacial de D. citri en Villa Altagracia y El Seibo. En Villa Altagracia, la media muestral obtenida para los huevos y ninfas fue menor que la varianza, por lo tanto el patrón de distribución para estos estados de la plaga es el agregado, esto fue confirmado al calcularse el Índice de Dispersión (ID), el Índice de Green (Cx), los valores de la media de Lloyd (m*) y el índice de Lloyd (L). En cada caso, los resultados de estos índices nos indican un patrón de distribución agregado para los huevos y las ninfas. Para los adultos, se encontró evidencias de que estos se distribuyen de manera uniforme en el cultivo, pues la media muestral fue mayor que la varianza. Esta uniformidad en el patrón espacial de los adultos, se pudo confirmar al calcular los demás índices de distribución espacial (Tabla I).

En El Seibo, los resultados obtenidos al aplicar los índices de dispersión a las poblaciones de huevos y ninfas mostraron evidencias de que estos tienden a distribuirse de manera uniforme. Contrario a esto, resultó que las poblaciones de los adultos se disponen de manera agregada en el cultivo (Tabla I).

Tabla I. Índices de agregación de D. citri obtenidos para Villa Altagracia y El Seibo.

\begin{tabular}{|c|c|c|c|c|c|c|c|}
\hline \multirow[b]{2}{*}{ Localidad } & \multirow[b]{2}{*}{ Estado de D. citri } & \multirow[b]{2}{*}{$\overline{\mathrm{X}}$} & \multirow[b]{2}{*}{$\mathrm{S} 2$} & \multicolumn{3}{|c|}{$\begin{array}{l}\text { Índices de } \\
\text { agregación }\end{array}$} & \multirow[b]{2}{*}{$\mathrm{Cx}$} \\
\hline & & & & ID & $\mathrm{m}^{*}$ & $\mathrm{~L}$ & \\
\hline \multirow{3}{*}{ VA } & Huevos & 2.56 & 4.4 & 1.71 & 3.4 & 1.32 & 0.01 \\
\hline & Ninfas & 2.88 & 5.8 & 2.01 & 3.89 & 1.02 & 0.01 \\
\hline & Adultos & 1.27 & 0.36 & 0.28 & 0.55 & 0.43 & -0.02 \\
\hline \multirow{3}{*}{ ES } & Huevos & 1.65 & 1.2 & 0.72 & 1.37 & 0.82 & -0.007 \\
\hline & Ninfas & 1.08 & 0.1 & 0.09 & 0.17 & 0.15 & -0.03 \\
\hline & Adultos & 2.04 & 2.26 & 1.1 & 2.14 & 1.04 & 0.002 \\
\hline
\end{tabular}

Leyenda: VA=Villa Altagracia; $\mathrm{ES}=\mathrm{El}$ Seibo; $\overline{\mathrm{X}}=$ Media muestral; $\mathrm{S}^{2}=$ Varianza; ID= Índice de dispersión; $\mathrm{m}^{*}=\mathrm{Media}$ de Lloyd; $\mathrm{L}=$ Índice de Lloyd; $\mathrm{Cx}=$ Índice de Green. 
Los diferentes patrones espaciales de $D$. citri encontrados en Villa Altagracia, probablemente se debieron a las densidades poblacionales de los adultos y la abundancia de brotes tiernos en el cultivo, los que podrían ser vitales para el desarrollo y alimentación de los inmaduros de D. citri, pudiendo influir la cantidad de brotes tiernos de un cultivo en las densidades poblacionales del psílido (Dharajothi et al., 1989; Tsai y Liu, 2000; Tsai et al., 2002; Halbert y Manjunath, 2004; Hall et al., 2008 y Qureshi et al., 2009). Además, en este campo aunque los árboles están afectados con el HLB y recibieron los efectos de un prolongado período de sequía durante nuestra investigación, se aplica un manejo constante del cultivo (riego y podas del dosel de los árboles constante, nutrición periódica de las plantas y el reemplazo de plantas viejas por jóvenes. Esto favoreció la producción y la alta densidad de brotes tiernos, creándose condiciones favorables para el incremento de las poblaciones de adultos de $D$. citri por todo el cultivo.

En El Seibo, los patrones espaciales de D. citri, también se vieron afectados por la abundancia de brotes tiernos presentes en los árboles. Aquí se observó menor densidad de brotes tiernos que en Villa Altagracia, debido probablemente a que las plantas en este campo están altamente afectadas por el HLB, no se aplica un manejo agronómico en este cultivo y a que la prolongada sequía pudo provocar que las plantas disminuyeran su capacidad de producir brotes. Estas condiciones desfavorables en todo el cultivo, pudo disminuir las densidades de adultos de D. citri y provocar que estos se desplazaran hacia otros árboles en busca de condiciones más favorables, promoviendo un patrón espacial agregado de los mismos.

Dinámica poblacional de D. citri en Villa Altagracia y El Seibo. Diaphorina citri estuvo presente durante todo el período de estudio en las localidades seleccionadas. En Villa Altagracia, las mayores densidades poblacionales de la plaga ocurrieron durante febrero, marzo, mayo y agosto (Fig. 1). En El Seibo, las poblaciones de adultos de D. citri fueron mayores durante abril y junio (Fig. 2).

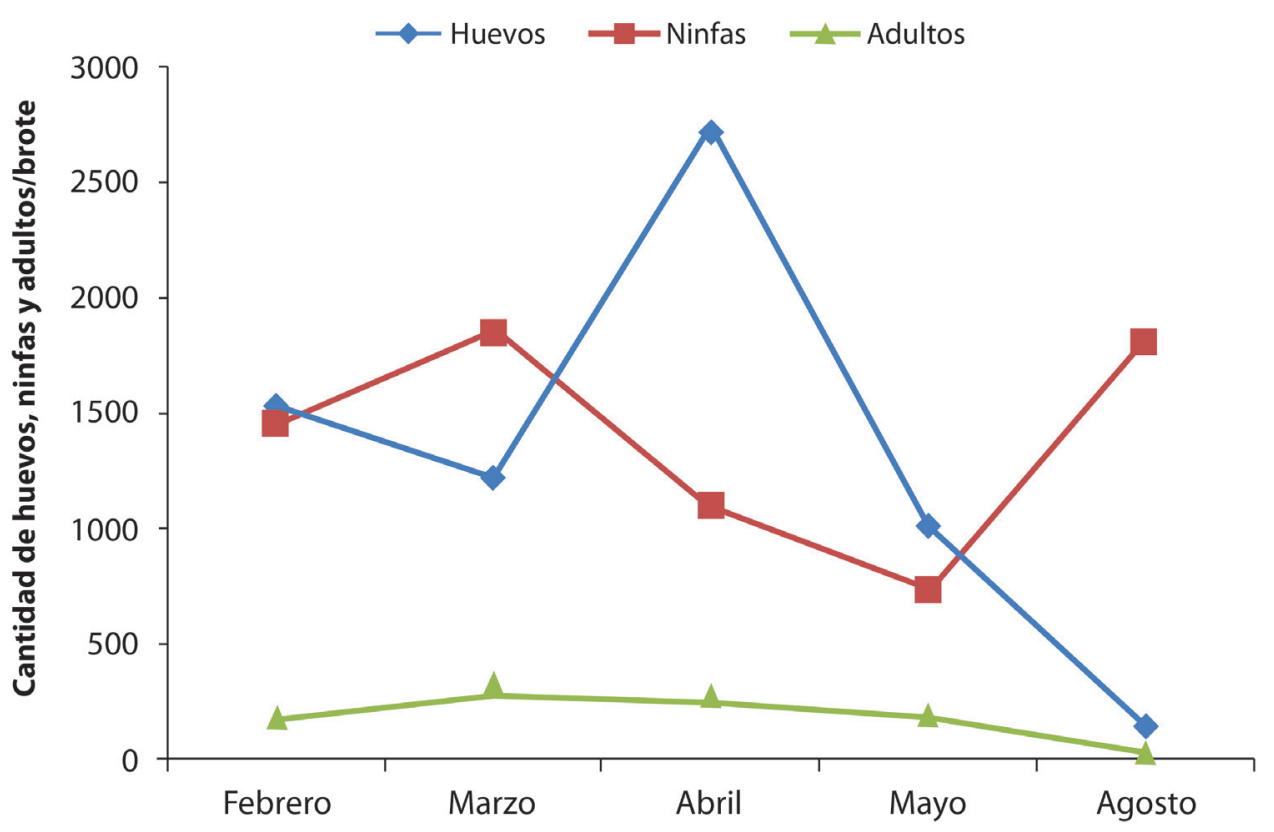

Figura 1. Fluctuación poblacional de D. citri por mes en Villa Altagracia, período febrero-agosto del 2013. 


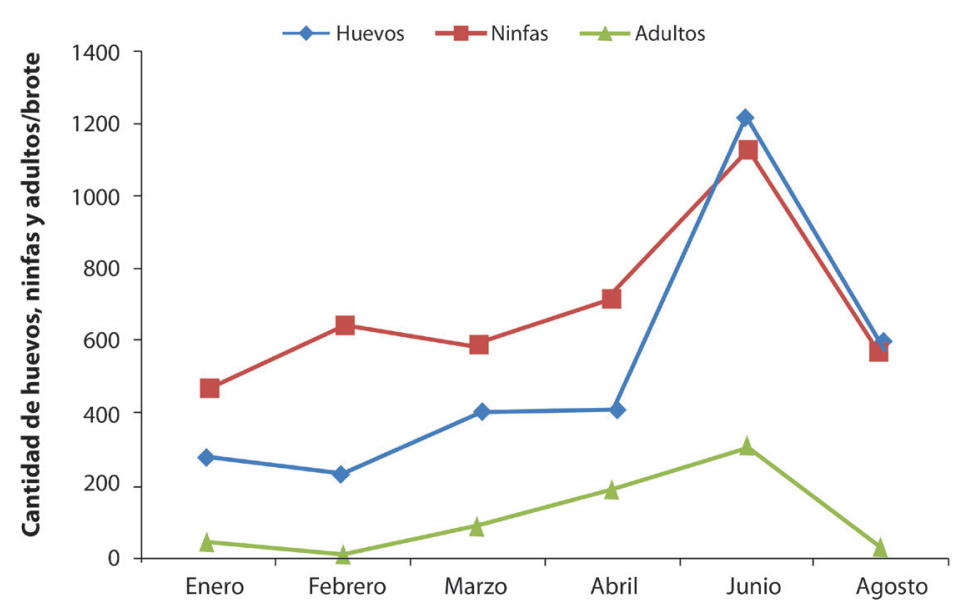

Figura 2. Fluctuación poblacional de D. citri por mes en El Seibo, período enero-agosto del 2013.

La dinámica de las poblaciones de $D$. citri registradas en Villa Altagracia y E1 Seibo fueron determinadas por la abundancia y disponibilidad de brotes tiernos; los picos poblacionales más altos de la plaga coincidieron con los momentos donde se observó mayor densidad de brotes tiernos en cada cultivo. Se encontraron diferencias altamente significativas $(p=0.0001)$ entre las densidades poblacionales de $D$. citri presentes en las localidades bajo estudio, registrando Villa Altagracia mayor cantidad de individuos de la plaga en comparación con El Seibo (Tabla II). Nuestros resultados concuerdan con los de Velásquez-Monreal et al. (2011) y López-López et al. (2011), quienes señalan que el manejo que se de en el cultivo puede afectar considerablemente las fluctuaciones y densidades poblacionales de la plaga.

Tabla II. Prueba de Kruskal Wallis: poblaciones de D. citri en Villa Altagracia y El Seibo.

\begin{tabular}{|c|c|c|c|c|c|c|c|c|}
\hline Local & Variable & $\mathrm{N}$ & Medias & D.E. & Medianas & $\begin{array}{l}\text { Promedio } \\
\text { de rangos }\end{array}$ & $\mathrm{H}$ & $\mathrm{P}$ \\
\hline $\mathrm{ES}$ & Huevos & 800 & 4.01 & 8.11 & 0 & 634.32 & 123.32 & $<0.0001$ \\
\hline $\begin{array}{l}\text { VA } \\
\text { Trat. }\end{array}$ & $\begin{array}{l}\text { Huevos } \\
\text { Rango }\end{array}$ & 700 & 9.48 & 15.31 & 4 & 883.28 & & \\
\hline ES & 634.32 & $\mathrm{~A}$ & & & & & & \\
\hline VA & 883.28 & B & & & & & & \\
\hline Local & Variable & $\mathrm{N}$ & Medias & D.E. & Medianas & $\begin{array}{l}\text { Promedio } \\
\text { de rangos }\end{array}$ & $\mathrm{H}$ & $\mathrm{P}$ \\
\hline $\mathrm{ES}$ & Ninfas & 800 & 5.12 & 6.56 & 3 & 634.12 & 123.75 & $<0.0001$ \\
\hline VA & Ninfas & 700 & 9.98 & 11.5 & 7 & 883.51 & & \\
\hline Trat. & Rango & & & & & & & \\
\hline $\begin{array}{l}\text { ES } \\
\text { VA }\end{array}$ & $\begin{array}{l}634.12 \\
883.51\end{array}$ & $\mathrm{~A}_{\mathrm{B}}$ & & & & & & \\
\hline Local & Variable & $\mathrm{N}$ & Medias & D.E. & Medianas & $\begin{array}{l}\text { Promedio } \\
\text { de rangos }\end{array}$ & $\mathrm{H}$ & $\mathrm{P}$ \\
\hline ES & Adultos & 800 & 0.84 & 1.13 & 1 & 659.88 & 75.03 & $<0.0001$ \\
\hline VA & Adultos & 700 & 1.29 & 1.41 & 1 & 854.06 & & \\
\hline Trat. & Rango & & & & & & & \\
\hline $\begin{array}{l}\text { ES } \\
\text { VA }\end{array}$ & $\begin{array}{l}659.88 \\
854.06\end{array}$ & $\mathrm{~A}_{\mathrm{B}}$ & & & & & & \\
\hline
\end{tabular}

Leyenda: VA= Villa Altagracia; ES= El Seibo; *letras distintas indican diferencias significativas $(\mathrm{p}<=0.05)$. 
Densidades de D. citri en la fenología del cultivo. En Villa Altagracia, los huevos fueron más abundantes en inicios de la floración (final de febrero); las ninfas registraron dos períodos con altas densidades poblacionales, a inicios de la floración (final de febrero) y durante el inicio de la brotación (inicios de agosto); por su parte, los adultos registraron mayor densidad y actividad a inicios de la floración (final de febrero) y a finales de la fructificación (final de mayo; Fig. 3). En El Seibo, durante el inicio de la brotación (final de junio), fue donde ocurrió mayor densidad de huevos y ninfas, mientras que el final de la fructificación (final de abril), mostró mayor densidad de adultos (Fig. 4).

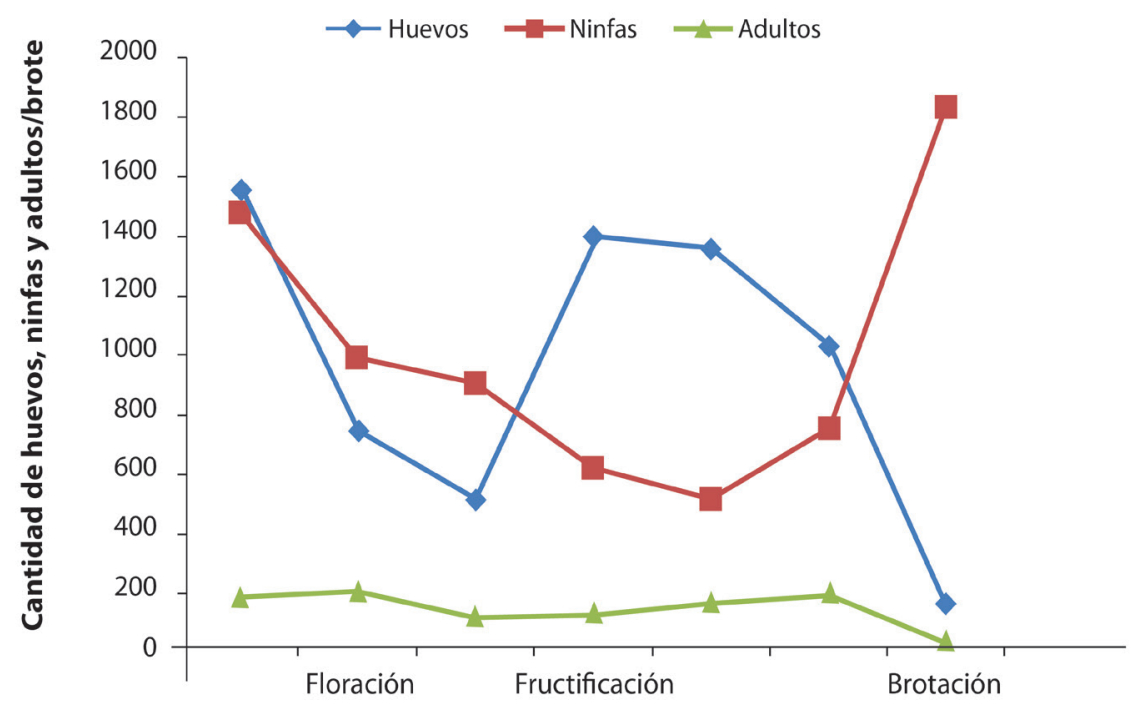

Figura 3. Fluctuación de D. citri en relación a la fenología del cultivo en Villa Altagracia, período febrero-agosto del 2013.

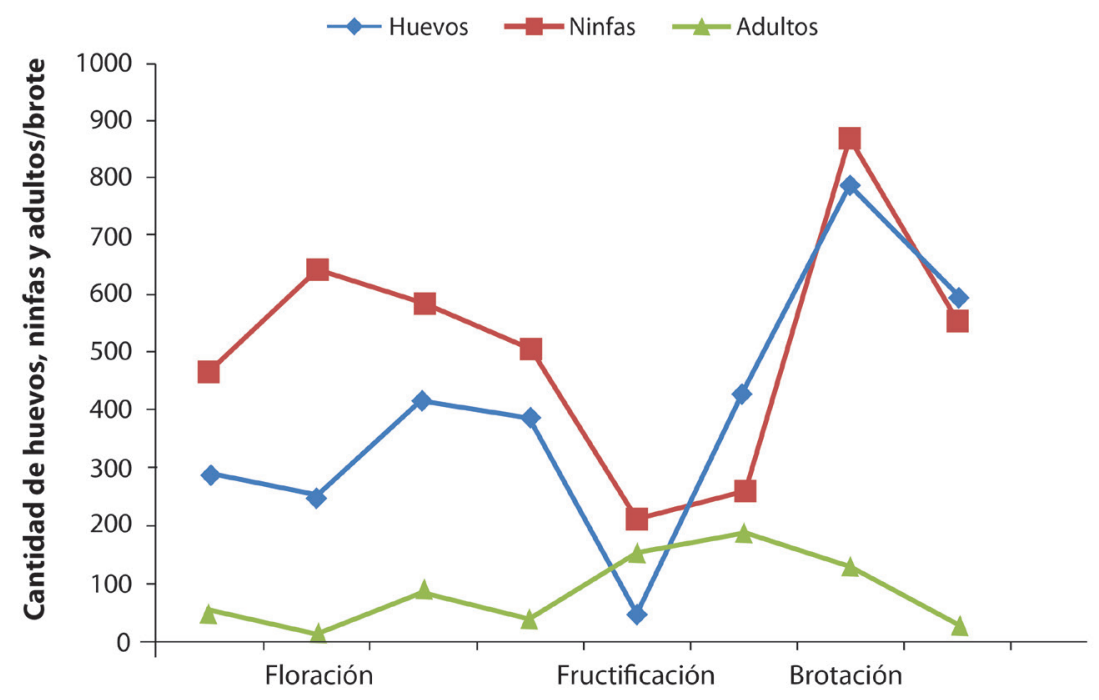

Figura 4. Fluctuación poblacional de D. citri en relación a la fenología del cultivo en El Seibo, período enero-agosto del 2013. 
Interacciones de D. citri con las condiciones climáticas. Se observó relación entre las densidades poblacionales de $D$. citri con las temperaturas y las lluvias registradas en Villa Altagracia y El Seibo (Tabla III). En Villa Altagracia, las poblaciones de ninfas de D. citri mostraron relación significativa y negativa (Prob $>\mathrm{R}=0.00005<\alpha=0.005$ ) con la temperatura y la lluvia, por su parte los adultos mostraron relación positiva (Prob $>R=0.00005<\alpha=0.005$ ) con la lluvia. En El Seibo, las poblaciones de adultos y huevos del psílido mostraron relación significativa y positiva (Prob $>\mathrm{R}=0.00005<\alpha=0.005$ ) con la temperatura y la lluvia, por su parte, las ninfas solo mostraron relación significativa y positiva (Prob $>R=0.00005<\alpha=0.005)$ con la lluvia.

Tabla III. Prueba de correlación de Spearman para D. citri, la temperatura y las lluvias en Villa Altagracia y El Seibo.

\begin{tabular}{|c|c|c|c|c|c|}
\hline \multicolumn{6}{|c|}{$\begin{array}{l}\text { Localidad = VA } \\
\text { Correlación Spearman: Coeficientes } \backslash \text { probabilidades }\end{array}$} \\
\hline & Huevos & Ninfas & Adultos & Temp $\mathrm{C}^{\circ}$ & Lluv (mm) \\
\hline Huevos & 1 & $1.60 \mathrm{E}-06$ & 0 & 0.98 & 0.58 \\
\hline Ninfas & 0.18 & 1 & 0.39 & 5.10E-07 & $3.40 \mathrm{E}-05$ \\
\hline Adultos & 0.36 & 0.03 & 1 & 0.42 & $2.10 \mathrm{E}-05$ \\
\hline Temp $\mathrm{C}^{\circ}$ & $1.00 \mathrm{E}-03$ & -0.19 & 0.03 & 1 & 0 \\
\hline Lluv (mm) & 0.02 & -0.16 & 0.16 & 0.41 & 1 \\
\hline \multicolumn{6}{|c|}{ Localidad $=$ ES } \\
\hline \multicolumn{6}{|c|}{ Correlación Spearman: Coeficientes\probabilidades } \\
\hline & Huevos & Ninfas & Adultos & Temp $\mathrm{C}^{\circ}$ & Lluv (mm) \\
\hline Huevos & 1 & 0 & 7.40E-09 & $1.50 \mathrm{E}-12$ & $4.00 \mathrm{E}-05$ \\
\hline Ninfas & 0.34 & 1 & 0.42 & 0.37 & $9.80 \mathrm{E}-07$ \\
\hline Adultos & 0.2 & -0.03 & 1 & $2.40 \mathrm{E}-04$ & $1.30 \mathrm{E}-03$ \\
\hline Temp $\mathrm{C}^{\circ}$ & 0.25 & 0.03 & 0.13 & 1 & 0 \\
\hline Lluv (mm) & 0.15 & 0.17 & 0.11 & -0.46 & 1 \\
\hline
\end{tabular}

Leyenda: VA= Villa Altagracia; ES=El Seibo.

El Análisis de Componentes Principales (ACP) en Villa Altagracia, mostró relación entre las poblaciones de ninfas de $D$. citri con la lluvia (Fig. 5); para esta localidad, el mes de agosto (período de brotación) fue el momento en que se registró menor pluviometría, favoreciendo a las ninfas del psílido; en el caso de los huevos y adultos de D. citri, se observó que en abril y mayo, fue el período de fructificación, la baja pluviometría fue favorable para estas fases de la plaga. Finalmente, la temperatura resultó ser independiente a las densidades poblacionales de D. citri, es decir, esta variable no tuvo incidencia sobre las poblaciones del psílido. En El Seibo, los huevos y ninfas de $D$. citri resultaron tener relación con la temperatura y la lluvia, esto ocurrió durante junio y agosto que fue el período de brotación, estos meses registraron niveles de pluviometría y temperatura favorables para estas fases de la plaga, registrándose mayor cantidad de los mismos en estos meses; por su parte los adultos del psílido mostraron independencia de la temperatura y la lluvia (Fig. 6). 


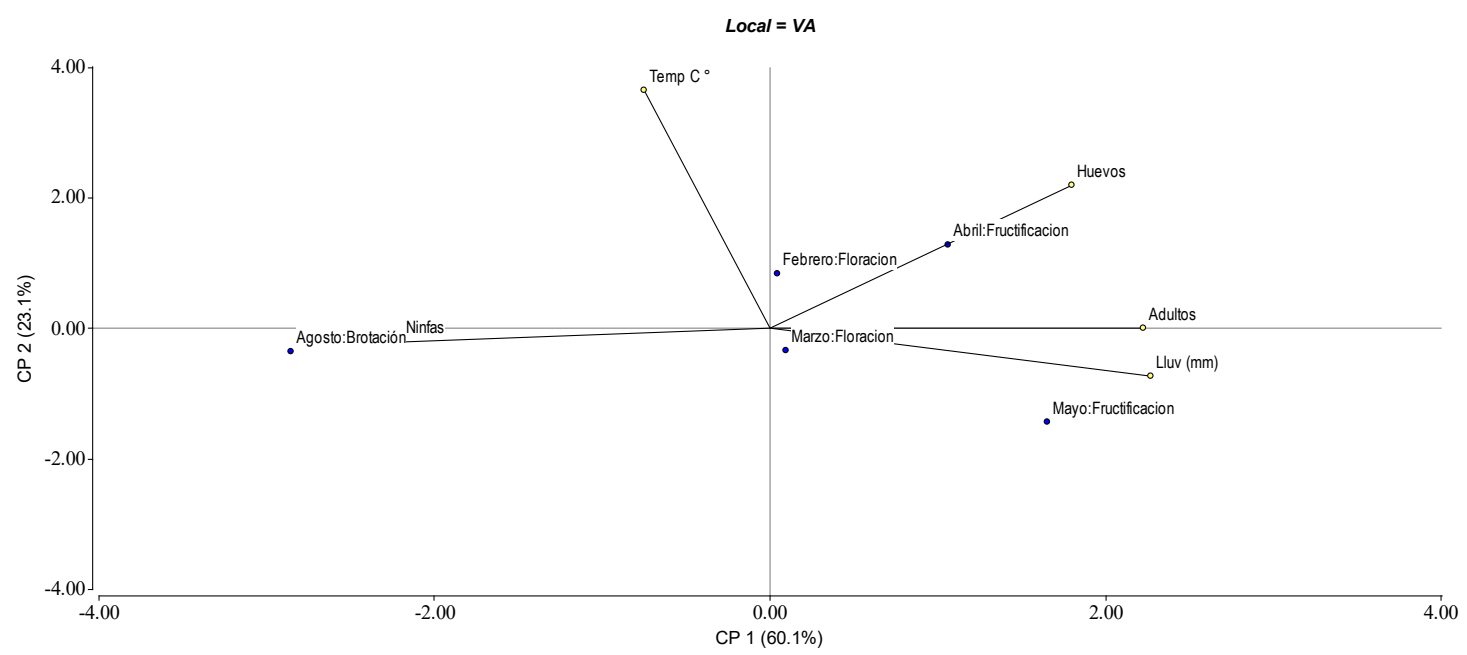

Figura 5. Análisis de Componentes Principales para D. citri en Villa Altagracia.

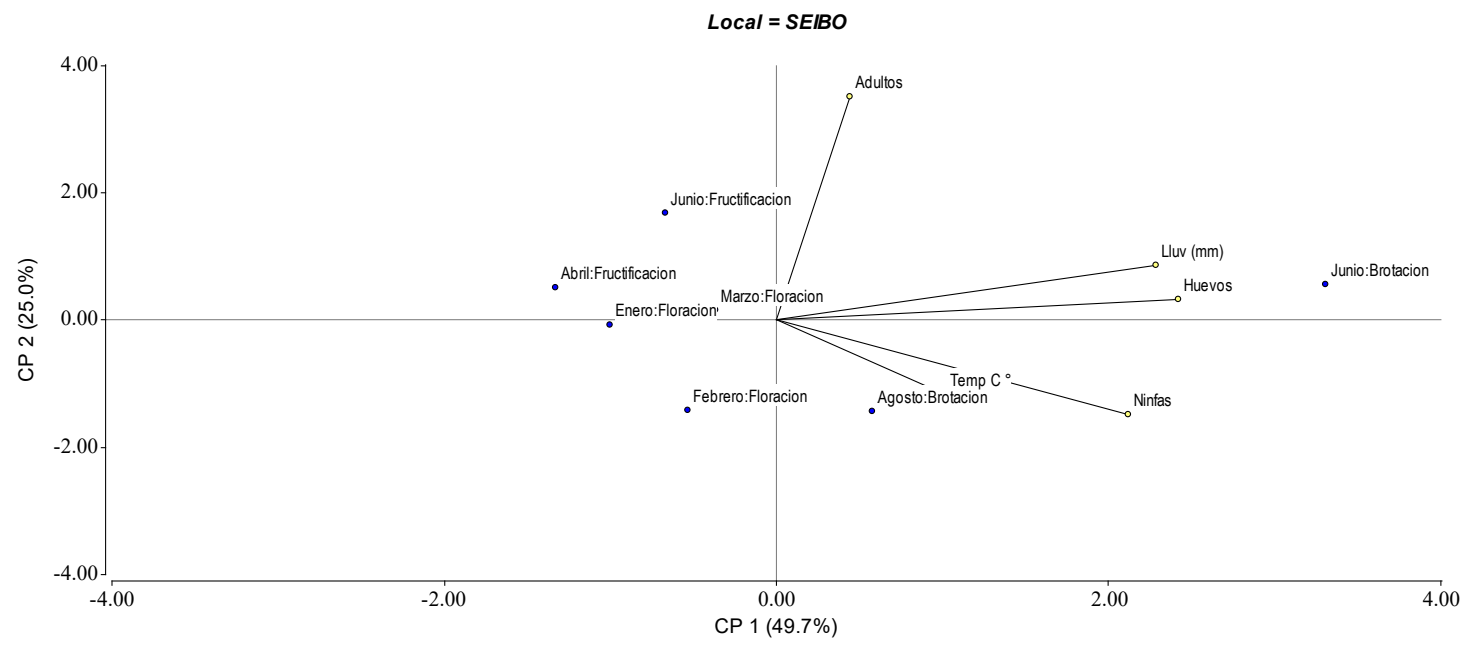

Figura 6. Análisis de Componentes Principales para D. citri en El Seibo.

Estos resultados son similares a las observaciones de Preza Durán (2011), quien detectó mayor actividad y densidad poblacional de $D$. citri durante la misma temporada que en nuestra investigación. Es bueno resaltar que algunos especialistas (Moschini et al., 2010; Preza Durán, 2011) indican que valores superiores de $150 \mathrm{~mm}$ de precipitación mensual provocan un gran impacto sobre las densidades poblacionales de $D$. citri, pues cuando las lluvias sobrepasan este rango pueden arrastrar huevos, ninfas o adultos del psílido de los brotes en que se encuentran, provocando una reducción en las poblaciones de esta plaga. Estas aseveraciones nos explican por qué las lluvias resultaron con tan poca incidencia sobre las poblaciones del psílido en Villa Altagracia y en El Seibo, pues para estas localidades las precipitaciones nunca sobrepasaron los $100 \mathrm{~mm}$ mensuales durante el transcurso de las observaciones, no siendo lo suficientemente fuertes como para arrastrar a cualquiera de las fases de desarrollo de $D$. citri de los brotes de los árboles. 


\section{AGRADECIMIENTOS}

Al personal del Instituto de Investigaciones Botánicas y Zoológicas Prof. Rafael M. Moscoso (IIBZ), por brindarnos todo su apoyo durante este trabajo de investigación. Al Consorcio Cítricos Dominicanos, en las personas del Felipe Méndez y Miguel A. Doñé (Chepe), por permitirnos la realización del trabajo en sus plantaciones. A José Páez Guerrero (Tito), por facilitarnos un lugar para poder realizar la investigación.

\section{LITERATURA CITADA}

Barrera, J. F., J. Herrera, F. Vásquez, M. Hernández, J. Gómez y J. Valle. 2011. Estudios sobre trampeo de Diaphorina citri (Hemiptera: Psyllidae): Trapping studies of Diaphorina citri (Hemiptera: Psyllidae). Inifap (Instituto Nacional de Investigaciones Forestales, Agrícolas y Pecuarias). $2^{\circ}$ Simposio Nacional sobre investigación para el manejo del Psílido Asiático de los Cítricos y el Huanglongbing en México, pp. 30-40.

Capoor, S. P., D. G. Rao y S. M. Viswanath. 1967. Diaphorina citri Kuwayama a vector of the greening disease of citrus in India. Indian Journal of Agricultural Science, 37: 572-575.

Cabrera Mireles, H. y F. D. Murillo Cuevas. 2010. Dinámica poblacional de Diaphorina citri (Hemiptera: Psyllidae) en la región Centro de Veracruz. INIFAP (Instituto Nacional de Investigaciones Forestales, Agrícolas y Pecuarias). México, DF. s. p.

Dharajothi, B., A. Verghese y P. L. Tandon. 1989. Ecological studies on citrus psylla, Diaphorina Citri Kuwayama (Hemiptera: Psyllidae) with special reference to its spatial distribution and sampling plan. Entomon, 14: 319-324.

French, J. V., C. J. Kahlke y J. V. da Graca. 2001. First record of the Asian citrus psylla, Diaphorina citri Kuwayama (Homoptera: Psyllidae), in Texas. Subtropical Plant Science, 53: 14-15.

García Dardares, C. 2009a. Diaphorina citri Kuwayama (Hemíptera: Psyllidae), vector de la bacteria que causa el Huanglongbing (HLB - Greening). Ministerio de la Producción Secretaría de Agricultura, Ganadería, Pesca y Alimentación Servicio Nacional de Sanidad y Calidad Agroalimentaria. Buenos Aires, AR., pp. 1-18.

García Darderes, C. 2009b. Distribución del Huanglongbing (HLB-Greening) en el Mundo. Ministerio de la Producción Secretaría de Agricultura, Ganadería, Pesca y Alimentación. Servicio Nacional de Sanidad y Calidad Agroalimentaria. Buenos Aires, AR. pp. 1-13.

Green, R. H. 1966. Measurement of non-randomness in spatial distributions Researches on Population Ecology, 8: 1-7.

Halbert, S. E. y K. E. Manjunath. 2004. Asian citrus psyllids (Sternorrhyncha: Psyllidae) and greening disease of citrus: A literature review and assessment of risk in Florida. Florida Entomologist, 87: 330-353.

Hall, D. G. 2009. An assessment of yellow sticky card traps as indicators of the abundance of adult Diaphorina citri (Hemiptera: Psyllidae) in citrus. Horticultural Research Laboratory, Subtropical Insects Research Unit, Florida (USA). Journal of Economic Entomology, 102: 446-452. 
Hall, D. G., M. G. Hentz y R. C. Adair Junior. 2008. Population ecology and phenology of Diaphorina citri (Hemiptera: Psyllidae) in two Florida citrus groves. Environmental Entomology, 37 (4): 914-924.

López-López, R., L. D. Ortega-Arenas, J. R. Lomelí-Flores, E. Cedillo-Portugal, L. GómezTovar y J. Salazar-Cruz. 2011. Abundancia de Diaphorina citri y entomófagos asociados en huertos citrícolas con diferentes sistemas de manejo de arvenses. 2do. Simposio Nacional sobre investigación para el manejo del Psílido Asiático de los Cítricos y el Huanglongbing en México. pp. 177-187.

Lloyd, M. 1967. Mean crowding. Journal of Animal Ecology, 36: 1-30.

Michaud, J. P. 2002. Biological control of Asian citrus psyllid, Diaphorina citri (Hemiptera: Psyllidae) in Florida: a preliminary report. Entomological News, Philadelphia, USA. Entomological News, 113: 216-222.

Moreno Pérez, M., E. Pozo Velásquez, R. Valdés Herrera y M. Cárdenas Morales. 2008. Distribución espacial de Diaphorina citri Kuwayama (Hemiptera: Psyllidae) sobre Lima Persa (Citrus latifolia Tanaka). Fitosanidad, 12: 33-37.

Morales, P. V., O. Fonseca, Y. Noguera, W. Cabaña, F. Ramos, E. Escalona, C. Rosales, M. Cermeli, B. Salas y E. Sandoval. 2010. Evaluación del ciclo de vida del psílido asiático de los cítricos en cinco plantas hospederas. Agronomía Tropical, 60: 238-286.

Moschini, R. C., G. E. Heit, H. A. Conti, G. Cazenave y P. L. Cortese. 2010. Riesgo agroclimático de las áreas citrícolas de Argentina en relación a la abundancia de Diaphorina citri. Alerta HLB Programa Nacional de Prevención del Huanglongbing. SENASA Informe de enero 2010. s. p.

Polek, M., V. Georgios y K. Godfrey. 2007. Citrus bacterial canker disease and Huanglongbing (Citrus greening). (en línea) Consultado 03 feb. 2012. Publication 8218 of The University of California, Division of Agricultural and Natural Resources. ARN Publication 8218. pp. 4-9. Disponible en http://anrcatalog.ucdavis.edu.

Preza Durán, A. 2011. Enemigos naturales de Diaphorina citri (Kuwayama) (Hemíptera: Psyllidae) en tres sitios del estado de Veracruz. Presentado en la Facultad de Ciencias Agrícolas de la Universidad Veracruzana, Diciembre 2011, MX. Tesis Ing. Agrónomo. $63 \mathrm{pp}$.

Qureshi, J. A. y P. A. Stansly. 2007. Integrated approaches for managing the Asian citrus psyllid Diaphorina citri (Hemiptera: Psyllidae) in Florida. Proceedings of the Florida State Horticultural Society, 120: 110-115.

Qureshi, J. A., M. E. Rogers, D. G. Hall y P. A. Stansly. 2009. Incidence of invasive Diaphorina citri (Hemiptera: Psyllidae) and its introduced parasitoid Tamarixia radiata (Hymenoptera: Eulophididae) in Florida citrus. Journal of Economic Entomology, 102: 247-256.

Ramos Méndez, C. 2008. Huanglongbing (“citrus greening”) y el psílido asiático de los cítricos, una perspectiva de su situación actual. Portal Agrosanitario, volumen?: 5-10. 
Robles-Gonzáles, M. M., J. J. Velázquez-Monreal, M. Orozco-Santos, M. A. Manzanilla Ramírez, R. Flores-Virgen, H. Arredondo-Bernal, A. B. Archila-Marroquín, M. C. Núñez-Camargo, M. Barba-Reynoso, J. G. Reyes-Martínez y J. I. Rodríguez-Acevedo. 2010. Bioecología del psílido asiático de los cítricos Diaphorina citri Kuwayama (Hemiptera: Psyllidae) en Limón Mexicano en Colima. VI. Simposio Internacional Citrícola. Tecoman, Colima, MX. pp. 31-73.

Sandoval-Rincón, J. A., S. A. Curti-Díaz, U. A. Díaz-Zorrilla, V. M. Medina-Urrutia y M. M. Robles-Gonzáles. 2010. Alternativas para el manejo del psílido Asiático de los cítricos (Diaphorina citri Kuwayama). Tecoman, Colima, MX. pp. 154-173.

Sétamou, M., D. Flores, J. V. French y D. G. Hall. 2008. Dispersion patterns and sampling plans for Diaphorina citri (Hemiptera: Psyllidae) in citrus. Journal of Economic Entomology, 101: 1478-1487.

Stansly, P. J. Arevalo y J. Qureshi. 2010. Monitoring methods for Asian citrus psyllid. Citrus Industry. (en línea). Consultado 20 feb. 2012. Disponible en http:/www.crec.ifas.ufl.edu/ extension/trade_journals/2010/2010\%20April\%20monitoring\%20psyllids.pdf.

Tsai, J. H. y Y. H. Liu. 2000. Biology of Diaphorina citri (Homoptera: Psyllidae) on four host plants. Journal of Economic Entomoly, 93: 1721-1725.

Tsai, J. H., J. J. Wang y Y. H. Liu. 2002. Seasonal abundance of the Asian citrus psyllid, Diaphorina citri (Homoptera: Psyllidae) in southern Florida. Florida Entomologist, 85: 446-450.

Toledo, J. y F. Infante. 2008. Manejo Integrado de Plagas, Capitulo 3: Aplicación de la Ley de Poder de Taylor. Primera Edición. Trillas México, D.F. p. 47-79.

Velázquez-Monreal, J. J., M. M. Robles-Gonzáles, M. A. Manzanilla-Ramírez, S. H. CarrilloMedrano y Orozco-Santos. 2011. Dinámica poblacional de Diaphorina citri Kuwayama (Hemiptera: Psyllidae) en el estado de Colima. 2do. Simposio Nacional sobre investigación para el manejo del Psílido Asiático de los Cítricos y el Huanglongbing en México-2011. pp .161-168.

Villalobos, W., D. Hollis, C. Godoy y C. Rivera. 2005. First report of Diaphorina citri (Hemiptera: Psyllidae) in Costa Rica. Insecta Mundi, 19: 191-192.

Yamamura, K. 1999. Transformation using $(\mathrm{x}+0.5)$ to stabilize the variance of populations. Researches on Population Ecology, 41: 229-234.

[Recibido: 22 de junio, 2016. Aceptado para publicación: 12 de septiembre, 2016] 